\title{
The pattern of responding after extensive extinction
}

\author{
PAULO GUILHARDI and RUSSELL M. CHURCH \\ Brown University, Providence, Rhode Island
}

\begin{abstract}
Extensive extinction greatly reduces response rate and increases the relative frequency of short interresponse times, but does not affect temporal learning or operant response rate. In each of two experiments, 24 rats were trained in a multiple cued interval procedure with three stimuli (noise, light, and clicker) at three intervals (30, 60, and $120 \mathrm{sec}$ ). In Experiment 1, after 50 sessions of extinction, response rate decreased from about 25 to 0.5 responses/min, but temporal discriminations were maintained and the initial response gradients in reacquisition had a pattern that corresponded with the original (rather than current) training conditions. In Experiment 2, these results were replicated and extended by examination of the effect of stimulus duration on response patterns during extinction, but its lack of effect on reacquisition. The similarity of the initial performance in reacquisition to the asymptotic performance in acquisition was presumably due to the similarity of context. The individual subject data may be downloaded from www.psychonomic.org/archive.
\end{abstract}

In temporal conditioning procedures, a reinforcer is delivered at a relatively constant interval after the onset of a stimulus. The pattern of behavior of an animal in such a procedure is usually characterized by an increase in the response rate as a function of time since the onset of the stimulus. This temporal gradient of responding provides information about the duration of the fixed interval: Responding typically begins at approximately a fixed proportion of the fixed interval (Schneider, 1969). In addition, overall response rate is positively related to the reinforcement rate (Gallistel \& Gibbon, 2000). Because the temporal gradient of responding can be decomposed into two parts (pattern and rate), it can be used for inferences about both the memory of interval duration and the memory of reinforcement rate (Kirkpatrick \& Church, 2003). In the Kirkpatrick and Church experiment, the distribution of times from stimulus onset to food delivery (fixed, random, or the sum of a fixed and a random time) determined the pattern of responding (increasing, flat, or flat followed by increasing patterns); the expected interval between stimulus onset and food (45, 90 , 180 , or $360 \mathrm{sec}$ ) determined the mean rate of responding.

After the training phase of a temporal conditioning procedure, a standard extinction procedure is one in which the conditions are identical to those during training, except that no reinforcers are delivered. The most obvious effect of such an extinction procedure is to reduce the amount of responding, but temporal discriminations remain. Examples include the extinction of a shuttlebox trace avoidance con-

This research was supported by National Institute of Mental Health Grant MH44234 to Brown University. The authors express appreciation to Mika MacInnis, Linlin Yi, and Marcelo Caetano for discussion about the experiments and manuscript. Correspondence should be addressed to P. Guilhardi, Department of Psychology, Box 1853, Brown University, Providence, RI 02912 (e-mail: paulo_guilhardi@brown.edu). ditioning performance in which the median latencies of an avoidance response of dogs were 1.9, 2.7, and $11.5 \mathrm{sec}$ for CS-US intervals of 5, 10, and $20 \mathrm{sec}$ (Kamin, 1954); and extinction of a classically conditioned heart rate response of dogs in which the mean latencies to the maximum response were 4.7 and $10.5 \mathrm{sec}$ for CS-US intervals of 5 and $20 \mathrm{sec}$ (Church \& Black, 1958). In both examples, the probability of a response decreased during extinction, but, if a response occurred, its latencies during extinction and acquisition were similar. Such results provided support for the principle that overall response probability decreases during extinction, but that response latency in extinction reflects the interval between stimulus and reinforcer in acquisition.

These results, based upon measures of central tendency, have been strengthened by experiments based on the mean response gradients in the peak procedure. Extinction reduced the mean response rate, but did not affect the time of maximum responding in an experiment on the extinction of rat lever pressing on a 40 -sec peak procedure (Roberts \& Holder, 1984, Experiment 5) and in an experiment on autoshaping of ring doves with CS-US intervals of 4, 8, and 16 sec (Ohyama, Gibbon, Deich, \& Balsam, 1999, Experiment 3). The latter experiment consisted of 120 presentations of the stimulus without reinforcement, which was sufficient to reduce the response rate greatly but maintain the relative peak gradients. Results of these experiments and others using fixed interval schedules, such as Machado and Cevik (1998), may have been restricted to the early stages of extinction and to the use of the same stimulus durations in extinction as in acquisition. The questions remain whether or not extensive extinction would have an influence on temporal information and whether or not this influence would be dependent on the similarity of stimulus durations during acquisition and during extinction. 
Because of the difficulty in assessing the temporal characteristics of response gradients from the low response rates that occur after extensive extinction, various transfer tests have been employed. Rapid relearning of the time interval in the first session of retraining (Roberts \& Holder, 1984 ) and the effect of changes in the CS-US interval in classical conditioning of the rabbit nictitating membrane response (Coleman \& Gormezano, 1971) have been used. They support the conclusion that interval discrimination developed in acquisition is maintained during extinction.

The conclusions from experiments on the effects of extinction in temporal conditioning procedures have been similar to conclusions from experiments on the effects of extinction of other learned behavior. Although the most obvious effect of the prolonged omission of reinforcement is a considerable decrease in response rate, it has been clear since Pavlov (1927), and from the major textbooks of the 1940s through the 1970s, that extinction does not eliminate the original learning (Hilgard \& Marquis, 1940; Hull, 1943; Kimble, 1961; Mackintosh, 1974). After extinction, there is considerable evidence for the retention of information that was acquired during original acquisition (see, e.g., Nevin, 1967, and many others). Further evidence from transfer designs have supported and extended this conclusion (Delamater, 1996; Rescorla, 1996, 2001).

The goal of the present experiments was to provide a description of both the rate and pattern of behavior during extensive extinction with stimulus durations that were consistent or inconsistent with the durations trained during acquisition. This would provide information about whether extinction affected only response rate, or whether it also had some influence on the content of temporal learning. Two methods of assessment were used: an assessment of behavior during extinction, and a comparison of the reacquisition under conditions that were consistent or inconsistent with previous training.

\section{EXPERIMENT 1}

In Experiment 1, rats were trained under three discriminative fixed intervals $(30,60$, and $120 \mathrm{sec})$ signaled by different stimuli (light, noise, and clicker). After training, the rats were exposed to extensive extinction in which no food was delivered following any of the head-entry responses during the three intervals. The pattern and rate of responding during the last sessions of extinction were used to assess directly the effects of extended extinction on the rate of responding and on the memory for interval duration.

After extinction, the rats were exposed to reacquisition of the temporal discrimination under conditions that were consistent or inconsistent with previous training. The question was whether the pattern of behavior during the initial cycles of reacquisition reflected the stimulus duration learned during acquisition rather than the stimulus duration during reacquisition. If the pattern of behavior was due to the treatment during acquisition, extinction affected the response rate but not the pattern of behavior learned in acquisition. Performance during extinction was accessed directly during extinction and indirectly during early stages of reacquisition.

\section{Method}

\section{Animals}

Twenty-four male Sprague Dawley rats (Taconic Laboratories, Germantown, NY) were used in the experiment. The rats were housed individually in a colony room on a 12:12-h light:dark cycle (lights off at 8:30 a.m.). The experimental sessions were conducted during the dark cycle, when rats are usually more active. Dim red lights provided illumination in the colony room and the testing room. The rats were fed a daily ration that consisted of 45-mg Noyes pellets (Improved Formula A) that were delivered during the experimental session, and an additional $15 \mathrm{~g}$ of FormuLab 5008 food given in the home cages after the daily sessions. Water was available ad libitum in both the home cages and experimental chambers. Providing the opportunity to drink during the sessions reduced satiation effects and potentially improved the temporal discrimination gradients. The rats arrived in the colony at about 35 days of age and were handled daily until the beginning of the experiment. The rats had training on a multiple cued interval procedure that began when they were 67 days old as described in Guilhardi and Church (2005). Training for the present experiment began when the rats were 166 days old.

\section{Apparatus}

The 12 boxes $(25 \times 30 \times 30 \mathrm{~cm})$ were located inside ventilated noise-attenuating enclosures $(74 \times 38 \times 60 \mathrm{~cm})$. Each box was equipped with a food cup and a water bottle. Three stimuli, referred to as noise, light, and clicker, were generated from modules from Med Associates, St. Albans, VT. The noise was a $70-\mathrm{dB}$ white noise, with an onset rise time and termination fall time of $10 \mathrm{msec}$, that was generated by an audio amplifier (Model ANL-926). The light was a diffused houselight (Model ENV-227M) rated to illuminate the entire chamber over $200 \mathrm{Lux}$ at a distance of 3 in. The clicker (Model ENV-135M) was a small relay mounted on the outside of the box that was used to produce an auditory click at a rate of $1 / \mathrm{sec}$. A pellet dispenser (Model ENV-203) delivered 45-mg Noyes (Improved Formula A) pellets into the food cup on the front wall. Each head entry into the food cup was detected by an LED photocell. A water bottle was mounted outside the box; water was available through a tube that protruded through a hole in the back wall of the box. Two Gateway Pentium III/500 computers running the Med-PC Medstate Notation Version 2.0 (Tatham \& Zurn, 1989) controlled the experimental events and recorded the time at which events occurred with a 2-msec resolution.

\section{Procedure}

Prior to each session, there was a test of each of the stimuli (noise, light, and clicker), the recording of licking, and the delivery of food pellets. There was one pellet in the food tray at the beginning of each session to reduce behavioral variability at the beginning of the session. The experiment consisted of 80 sessions divided into three phases (acquisition, extinction, and reacquisition). Each of the daily sessions consisted of 60 cycles, as described below.

Phase 1: Acquisition (Sessions 1-20). In the acquisition phase, all rats were trained under 30-, 60-, and 120-sec fixed interval schedules of reinforcement differentially signaled by white noise, light, or clicker. This was a multiple cued interval (MCI) temporal discrimination procedure. A cycle of the MCI procedure consisted of a 20 -sec period during which the discriminative stimulus was off, a fixed interval with the discriminative stimulus on, and food primed at the end of the fixed interval. The next head entry into the food cup (measured as the time of the breaking of a photo beam in the food cup) produced the delivery of food, the termination of the discriminative stimulus, and the beginning of the next cycle. There were six (3!) possible combinations of stimulus and interval, and 4 rats were randomly assigned to each combination. For example, a particular 
rat had a 30 -sec interval signaled by white noise, a 60 -sec interval signaled by light, and a 120 -sec interval signaled by the clicker. The stimulus terminated and food was delivered following the first headentry response after the fixed interval. The assignment of stimuli to intervals was counterbalanced across animals and was consistent with their previous training. One of the three possible intervals (30, 60 , and $120 \mathrm{sec}$ ) was presented randomly (with replacement) with equal probability on each cycle of a daily session that consisted of 60 cycles. After 20 sessions of training, each rat received approximately 400 cycles of each interval.

Phase 2: Extinction (Sessions 1-50). In the extinction phase, the procedure was the same as that for acquisition, except that, at the end of the fixed interval, the stimulus was turned off and no food was delivered; head-entry responses into the food cup were recorded, but they had no consequences.

Phase 3: Reacquisition (Sessions 1-10). The procedure in the reacquisition phase consisted of the delivery of reinforcement following the first head entry into the food cup after the 30-, 60-, or 120 -sec interval. The 4 rats that were trained with each of the six combinations of stimulus and interval were randomly partitioned into two groups. Thus, 12 rats were assigned to the consistent group, and 12 were assigned to the inconsistent group. In the consistent group, each of the three fixed intervals $(30,60$, and $120 \mathrm{sec})$ was retrained with the same discriminative stimulus used in the first phase; in the inconsistent group, each of the three fixed intervals was retrained with a different discriminative stimulus. For example, a rat that had noise, light, and clicker associated with the fixed intervals 30,60 , and $120 \mathrm{sec}$, respectively, might have the noise associated with the 60 -sec fixed interval, the light associated with the 120 -sec fixed interval, and the clicker associated with the 30 -sec fixed interval. The stimulus used for each interval was counterbalanced across rats.

The letter "A" refers to one of the six possible orders of assignment of stimuli to intervals during acquisition (e.g., noise for FI $30 \mathrm{sec}$, light for FI $60 \mathrm{sec}$, and clicker for FI $120 \mathrm{sec}$ ), and the letter "B" refers to one of the two possible alternatives in which all stimuli were paired with different fixed intervals (e.g., noise for FI $60 \mathrm{sec}$, light for FI $120 \mathrm{sec}$, and clicker for FI $30 \mathrm{sec}$, or noise for FI $120 \mathrm{sec}$, light for FI $30 \mathrm{sec}$, and clicker for FI $60 \mathrm{sec}$ ). Group AAA refers to the rats in the consistent group that were presented with the same assignment of stimuli to intervals during acquisition, extinction, and reacquisition (A), and Group $A A B$ refers to the rats in the inconsistent group that were presented with a different assignment of stimuli to intervals during reacquisition (B). The letter $\mathrm{X}$ will be used to refer to either $\mathrm{A}$ or $\mathrm{B}$, so that all rats in Experiment 1 received condition AAX in the three phases.

\section{Data Analysis}

Overall response rate. The mean response rate for each rat during the stimulus-on period as a function of sessions of training was used to establish an acquisition baseline, and to investigate the effects of extinction on response rate.

Response pattern. Response rate as a function of the time since stimulus onset was calculated for each rat and served as an absolute measure of response pattern. These response rate gradients were fitted by a three-parameter ogive function:

$$
y=\frac{c}{1+e^{-(t-a) / b}}, \quad c \geq 0,
$$

with $a$ as an estimate of the center (the time at which response rate reached half of the way to its estimated maximum response rate), $b$ as an estimate of the scale of the function, $c$ as an estimate of the maximum response rate of the function, and $t$ the time since stimulus onset. The best-fitting three-parameter ogives were obtained with a nonlinear search algorithm that minimized the sum of squared deviations from the mean rate. This was done with the nlinfit function of MATLAB (The MathWorks, Natick, MA).

Proportion agreement. This measure was designed to determine which experimental phase best predicted the performance on a particular phase. For example, the performance during an early stage of reacquisition might be best predicted by the conditions of acquisition, but, during a later stage of reacquisition, it might be best predicted by the conditions of reacquisition. Proportion agreement was a measure of the similarity of the observed and expected behavior of each rat during selected stages of reacquisition.

The observed measure of behavior was calculated as follows: (1) The mean response rate as a function of time since stimulus onset was calculated for the three stimulus durations for each rat over selected sessions; (2) this absolute response gradient was divided by the maximum response rate to obtain a mean relative response rate; and (3) the order of the mean relative response rate was determined during the first $30 \mathrm{sec}$ of each of the stimuli. Thus, the observed order would be some permutation of the numbers 1,2 , and 3 .

If the observed behavior was determined by the treatment on a particular phase of training, the expected order of the mean relative response rate during the first $30 \mathrm{sec}$ was that it would be inversely related to the duration of the interval. The discrepancy $(D)$ between the observed order $O_{i}$ (e.g., 213 for the 30-, 60-, and 120-sec intervals, respectively) and expected $E_{i}$ order (e.g., 123 for the 30-, 60-, and 120 -sec intervals, respectively) was defined as

$$
D=\sum_{i=1}^{n}\left(O_{i}-E_{i}\right)^{2}
$$

The proportion agreement was defined as

$$
P=1-\frac{D}{m},
$$

where $P$ is the proportion agreement and $m$ is the maximum of the discrepancy between any possible observed order and the expected order determined by the treatment on a particular phase of training. Thus, the proportion agreement was equal to one minus the discrepancy relative to the maximum discrepancy. The proportion agreement varies from 0 to 1 with a chance value of .5 .

Bout analysis. The relative frequency distribution of the interresponse times less than $2.5 \mathrm{sec}$ during the three stimulus intervals $(30,60$, and $120 \mathrm{sec})$ provided a description of the clusters of responses that are referred to as bouts.

\section{Results \\ Acquisition and Extinction}

The effects of stimulus duration $(30,60$, and $120 \mathrm{sec})$ on the overall response rate during acquisition are shown in the left side of the top panel of Figure 1. The overall response rate during the last 10 sessions of acquisition (Sessions 11-20) was inversely related to the interval duration in acquisition. The mean overall response rates during the last 10 sessions of acquisition were 30.3, 26.2, and 19.0 responses/min during the 30-, 60-, and 120-sec intervals, respectively, and were related to interval duration $[F(2,46)=23.68, p<.001]$.

The effects of extinction on the overall response rate are shown in the right side of the top panel of Figure 1. The overall response rates during the 30-, 60-, and 120-sec stimuli decreased from the rates at the end of acquisition to less than $1 \mathrm{response} / \mathrm{min}$. The effects of stimulus duration $(30,60$, and $120 \mathrm{sec}$ ) on the overall response rate during later sessions in extinction are shown in the bottom panel of Figure 1 on a scale of $0-1$ response/min. The overall response rate averaged across the last 25 sessions of extinction as a function of stimulus duration were $0.61,0.52$, and 0.40 response $/ \mathrm{min}$ during the 30-, 60-, and 120-sec stimuli, respectively, and they were related to stimulus duration during extinction $[F(2,46)=4.86, p=.012]$. 

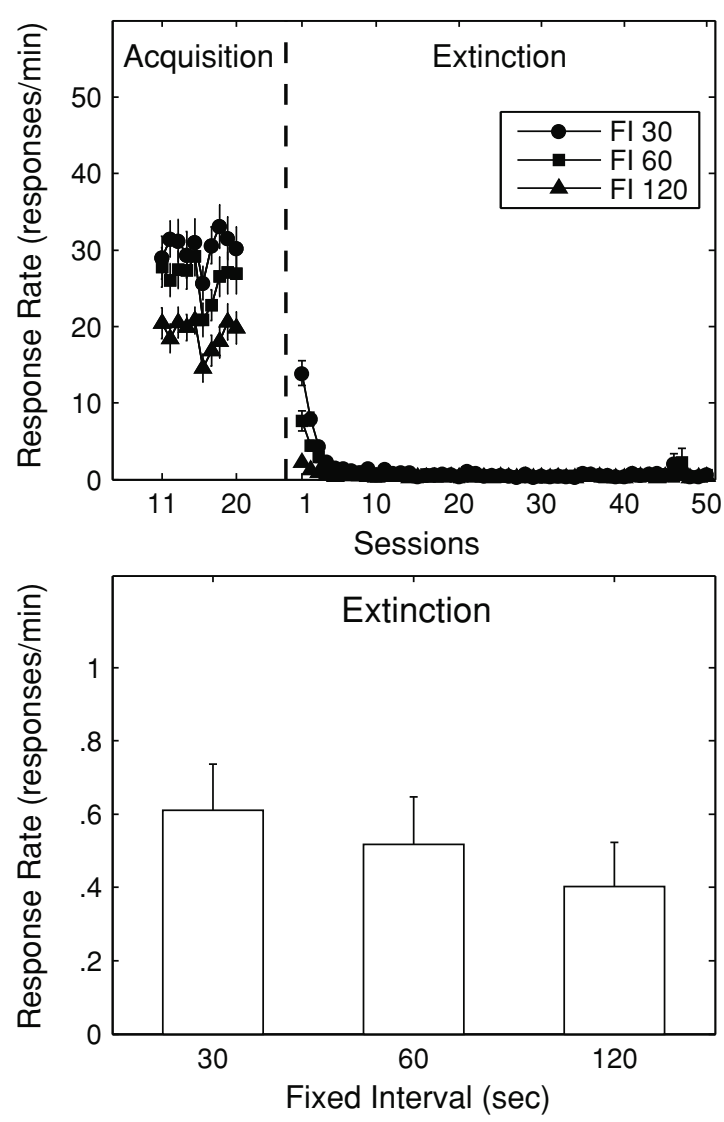

Figure 1. Top: Overall response rate during the stimuli as a function of sessions during the last 10 sessions of acquisition and during the 50 sessions of extinction during the fixed 30-, 60-, and 120 -sec intervals. Bottom: Overall response rate averaged across the last $\mathbf{2 5}$ sessions of extinction as a function of fixed interval. The response rate scale in the bottom panel is $2 \%$ of the scale in the top panel.

The effects of the stimulus duration (30,60, and $120 \mathrm{sec})$ on the pattern of responses during the last half of the sessions during acquisition are shown in the top panel of Figure 2. The data are represented as the means across individual rats. The mean response rate as a function of stimulus onset during the last 10 sessions of acquisition (Sessions 11-20) was approximately an ogive function. Although response rate is shown in absolute, rather than relative, units, the center and scale parameters of the ogive function were indicative of the pattern of responses. The proportion of variance accounted for by the ogive functions averaged across rats and stimulus durations was .985 , with an interquartile range of .005 . The centers of the individual ogive functions were positively related to stimulus duration $[F(2,46)=1,716.5$, $p<.001]$ (means of 24.2, 48.7, and 98.2 at 30-, 60-, and 120 -sec intervals, respectively). The scales of the individual ogive functions were also positively related to the stimulus duration $[F(2,46)=274.5, p<.001]$ (means of 4.6, 8.7, and 16.43 at 30-, 60-, and 120 -sec stimuli, respectively).

The response rate gradients during the early cycles in extinction were related to the stimulus duration in extinc- tion. The magnitude of the increase of the response rate functions of the individual rats was estimated by the slopes of a linear fit to the individual data. The mean slopes of the response rate gradients during the early cycles (Session 1) in extinction were $57.4,16.1$, and 1.6 for the $30-$, $60-$, and 120 -sec stimuli. These slopes were greater than zero $[t(23)=8.39, p<.001]$ and were related to stimulus duration in extinction $[F(2,46)=37.71, p<.001]$.

The response rate gradients during later sessions in extinction (last 25 sessions) were also related to the stimulus duration in extinction as shown in the bottom panel of Figure 2. The lines shown in the averaged data are the best-fitting ogive (Equation 1) to the data. The magnitude of the increase of the response rate functions of the individual rats was estimated by the slopes of a linear fit to the individual data. These slopes were greater than zero $[t(23)=5.93, p<.001]$ and were also related to the stimulus duration $[F(2,46)=12.18, p<.001]$. The slopes for individual rats and mean across rats during the 30-, 60-, and 120 -sec stimuli are shown in Table 1 for all rats, since all rats had the same assignment of intervals to stimuli in extinction that they had in acquisition (AAX).

The ogival functions characteristic of the response patterns during acquisition and extinction started at an ini-
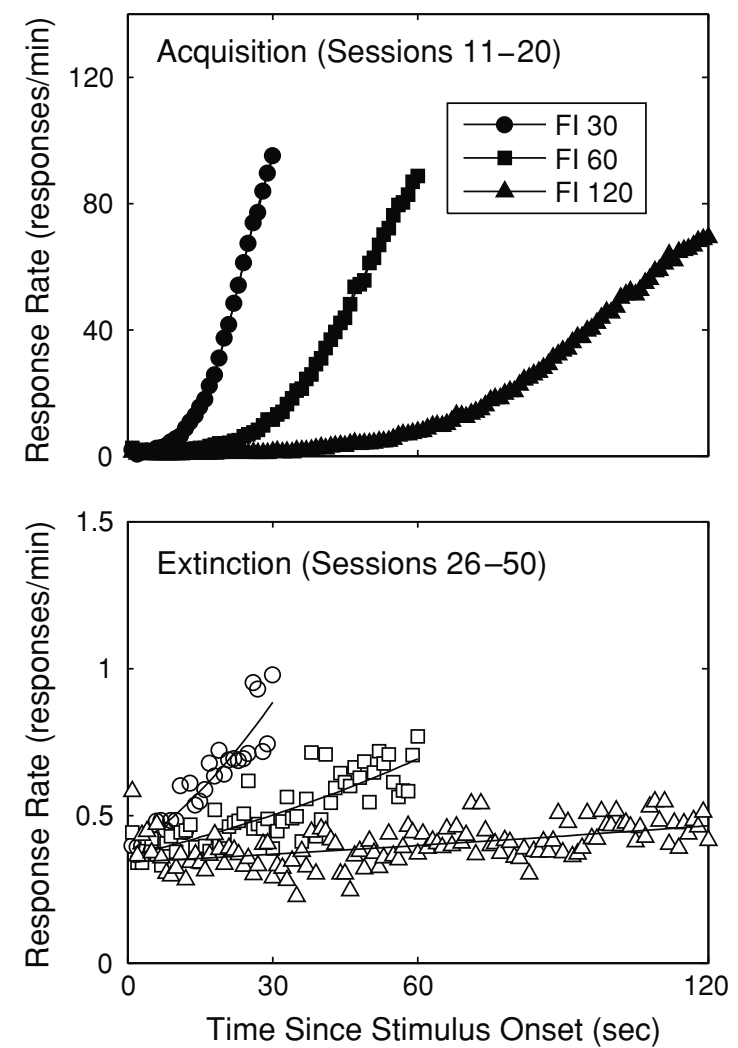

Figure 2. Response rate as a function of time since stimulus onset during the last 10 sessions of acquisition (top panel) and last 25 sessions of extinction (bottom panel) during the fixed 30-, 60-, and 120-sec intervals. The lines are the best-fitting ogives (Equation 1) to the data. 
Table 1

Slopes of a Linear Fit (Least Squares Criterion) to the Response Gradients of Individual Rats During the Last Half of Sessions of Extinction of Experiment 1 (Sessions 26-50)

\begin{tabular}{|c|c|c|c|}
\hline \multirow[b]{2}{*}{ Rats } & \multicolumn{3}{|c|}{ Fixed Intervals (sec) } \\
\hline & 30 & 60 & 120 \\
\hline 1380 & 1.15 & 0.25 & 0.23 \\
\hline 1381 & 1.36 & 0.37 & 0.00 \\
\hline 1383 & 2.19 & 0.08 & 0.00 \\
\hline 1385 & -0.26 & -0.06 & 0.15 \\
\hline 1390 & -0.04 & 0.42 & -0.04 \\
\hline 2371 & 0.29 & 0.03 & 0.06 \\
\hline 2374 & 0.40 & -0.01 & 0.01 \\
\hline 2375 & -0.26 & 0.10 & 0.12 \\
\hline 2376 & 0.67 & 1.11 & 0.06 \\
\hline 2382 & 1.00 & -0.04 & 0.11 \\
\hline 2386 & 1.54 & 0.34 & -0.02 \\
\hline 2388 & 1.38 & 0.83 & -0.02 \\
\hline 1370 & -0.62 & 1.09 & 0.29 \\
\hline 1373 & 0.79 & -0.03 & 0.05 \\
\hline 1379 & 1.72 & 0.65 & 0.05 \\
\hline 1384 & 1.18 & 0.21 & -0.06 \\
\hline 1387 & 1.48 & -0.23 & 0.04 \\
\hline 1389 & 0.08 & 0.12 & 0.19 \\
\hline 1391 & 2.07 & 0.09 & 0.03 \\
\hline 2368 & 0.21 & -0.01 & 0.08 \\
\hline 2369 & 0.19 & 0.91 & -0.01 \\
\hline 2372 & 0.92 & 0.55 & -0.07 \\
\hline 2377 & 4.44 & 0.03 & 0.20 \\
\hline 2378 & 2.86 & 1.08 & 0.00 \\
\hline Mean & 1.03 & 0.33 & 0.06 \\
\hline
\end{tabular}

tial rate greater than zero during both acquisition and extinction. This initial operant response rate was measured as the rate during the first second after stimulus onset. It was not related to the stimulus duration $[F(2,46)=$ $1.127, p=.333]$, it was similar during acquisition and extinction $[F(1,23)=1.635, p=.214]$, and there was no stimulus duration $\times$ reinforcement condition interaction $[F(2,46)=1.928, p=.157]$. The mean initial rates during acquisition were $2.15,2.49$, and 1.34 for the $30-, 60-$, and 120 -sec stimuli, respectively, and during extinction were $0.39,0.43$, and 0.57 for the $30-, 60-$, and 120 -sec stimuli, respectively.

The decrease in overall response rate within a session during extinction is shown in Figure 3. The data are the mean response rates averaged across stimulus durations and rats during the last 25 sessions of extinction. Although there was a mean of 20 cycles of each stimulus duration (of a total of 60 cycles), in some sessions there were stimulus durations with fewer than 20 cycles. Thus, data in Figure 3 are presented for the first 15 cycles of each stimulus duration. The response rate was higher in Cycle 1 than in Cycle $15[t(23)=5.77, p<.001]$. Although response rate at the beginning of a session was greater than response rate at the end of the session, responses still occurred later in a session. The response rates during Cycles 11-15 of the last 25 sessions of extinction were similar at all stimulus durations: The response rates were 0.49 , 0.31 , and 0.25 response/min, and the standard errors of the means were $0.21,0.08$, and 0.07 , for the fixed $30-, 60-$, and 120 -sec intervals, respectively.
The interresponse time (IRT) distributions were not affected by the stimulus durations but were affected by extinction. The relative frequencies of IRTs ranging from 0 to $2.5 \mathrm{sec}$ during the last 10 sessions of acquisition and last 25 sessions of extinction are shown in Figure 4. The means of the modes of the IRT distributions across rats were shorter during extinction than during acquisition $[t(23)=4.73, p<.001]$. The mean modes of the IRT distributions averaged across rats and stimulus durations were $0.28 \mathrm{sec}$ during acquisition and $0.17 \mathrm{sec}$ during extinction, with the standard errors of the means approximately $0.02 \mathrm{sec}$ during both acquisition and extinction. Thus, the IRTs were about $0.11 \mathrm{sec}$ longer during acquisition than during extinction.

During both acquisition and extinction, the IRTs from 0 to $2.5 \mathrm{sec}$ clustered below $0.5 \mathrm{sec}$. During acquisition, these short IRTs accounted for $90.4 \%, 90.3 \%$, and $86.2 \%$ of the IRTs in the 30-, 60-, and 120-sec intervals, respectively; during extinction, these short IRTs accounted for $63.8 \%, 56.1 \%$, and $52.9 \%$ of the IRTs in the $30-, 60-$, and 120 -sec intervals, respectively. During acquisition, the standard errors of the means were $0.86 \%, 0.93 \%$, and $1.57 \%$ in the $30-, 60-$, and 120 -sec intervals, respectively; during extinction, they were $2.45 \%, 2.44 \%$, and $2.81 \%$, in the 30-, 60-, and 120-sec intervals, respectively. The longer IRTs during acquisition were quite distinct from the shorter IRTs during extinction.

\section{Reacquisition}

The overall response rate during the stimulus in reacquisition rapidly increased from the low rate at the end of extinction to a rate similar to the rate at the end of acquisition. The overall rates early in reacquisition (Cycles 1-3) as a function of the stimulus duration during acquisition were 41.4, 41.3, and 25.7 responses/min for Group AAA, and 55.0, 33.0, and 15.9 responses/min for Group AAB, for the $30-, 60-$, and 120 -sec intervals. There was a main effect of stimulus duration $[F(2,44)=18.45, p<.001]$,

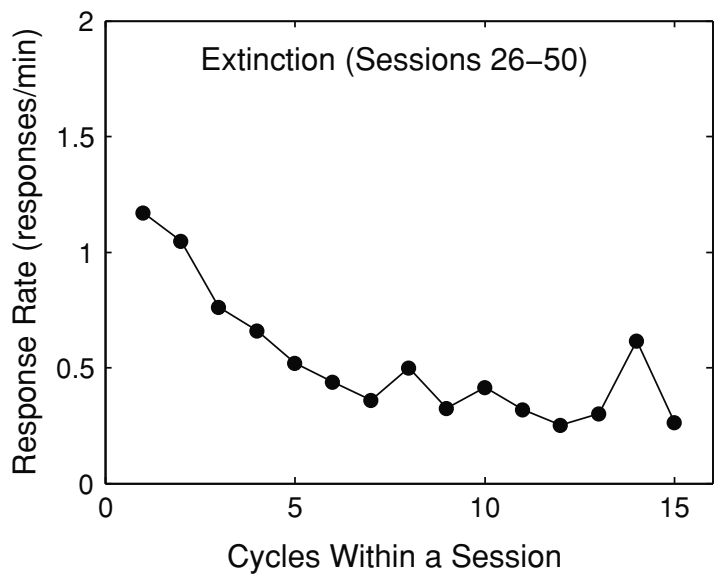

Figure 3. Response rate as a function of the first 15 cycles within a session averaged over the last 25 sessions of extinction and averaged across the fixed 30-, 60-, and 120-sec intervals. 


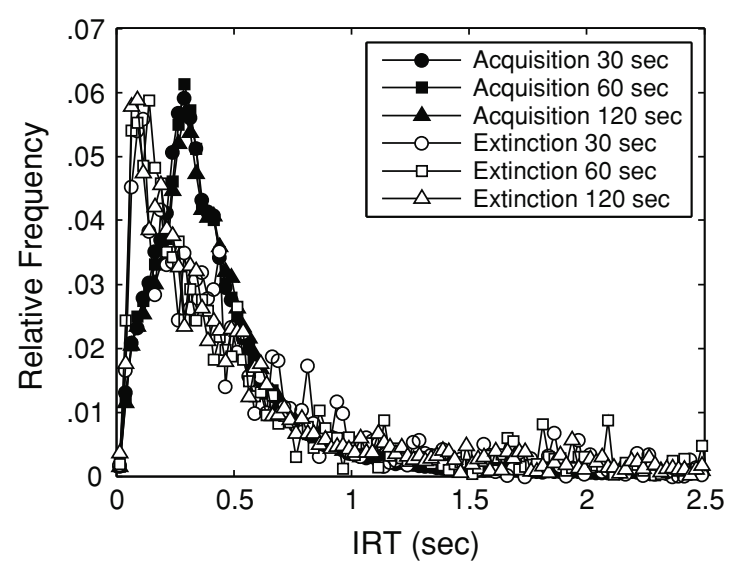

Figure 4. Relative frequency of interresponse intervals (IRTs) ranging from 0 to $2.5 \mathrm{sec}$ during the last 10 sessions of acquisition (filled symbols) and last 10 sessions of extinction (open symbols) during the fixed 30-, 60-, and 120-sec intervals.

no effect of group $[F(1,22)=0.086, p=.773]$, and a stimulus duration $\times$ group interaction $[F(2,44)=9.10$, $p<.01]$. Early in reacquisition (Cycles 1-3), the rates of responses for the different stimulus durations were similar to the rates during acquisition. The response rate order was inversely related to the stimulus duration presented during acquisition and reacquisition for Group AAA, but only inversely related to the stimulus duration presented during acquisition for Group AAB.

The response rate order during the early cycles in reacquisition (Cycles 1-3) was inversely related to the interval duration during reacquisition for Group AAA, but not for Group AAB. There was no effect of stimulus duration $[F(1,22)=2.83, p=.070]$ or of group $[F(1,22)=0.086$, $p=.773$ ], but there was a stimulus duration $\times$ group interaction $[F(2,44)=13.77, p<.001]$.

The response rate gradients during the first three cycles, first session, and last session of reacquisition are shown in Figure 5 in comparison with the response rate gradients during Sessions 11-20 of acquisition. During reacquisition, the response gradients were related to the stimulus durations 30, 60, and $120 \mathrm{sec}$ (Figure 5, empty symbols) and approximated the gradients during acquisition performance (Figure 5, filled symbols). This is shown for the first three cycles (top panel), for the first session (middle panel), and for the last session (bottom panel) of reacquisition for Group AAA. In all cases, the reacquisition gradients were similar to the acquisition gradients. The similarity of the gradients was even apparent during the first three cycles of reacquisition (top panel).

The response rate gradients during reacquisition are also shown in Figure 6 for both Group AAA (in which the "From interval" and the "To interval" are the same durations) and Group AAB (in which the "From interval" and the "To interval" are different durations). During the first three cycles (Figure 6, top row of panels) and during the first session of reacquisition (Figure 6, center row of panels), the response gradients were related to the stimulus durations trained during acquisition (and/or extinction), not to the intervals being trained in reacquisition. In contrast, during the last session of reacquisition (Figure 6, bottom row of panels), the response gradients were related to the stimulus durations trained during reacquisition, but not related to the stimulus durations trained during acquisition and extinction in Group AAB.

During the first $30 \mathrm{sec}$ of each stimulus (30,60, and $120 \mathrm{sec})$, the relative response rate was inversely related to the stimulus durations. (See the first $30 \mathrm{sec}$ of gradients in Figure 2, as well as the description of the "proportion agreement" measure in the data analysis section of this experiment.) Figure 7 shows the proportions of agreement of the observed order of the relative response rate during the first $30 \mathrm{sec}$ of the $30-, 60-$, and 120 -sec stimuli with the expected order, based on the assignments of stimuli to intervals used in original acquisition (A) or on the assignments of stimuli to intervals used in reacquisition in Group AAB (B). The horizontal axis is the amount of reacquisition training (first three cycles, first session, and
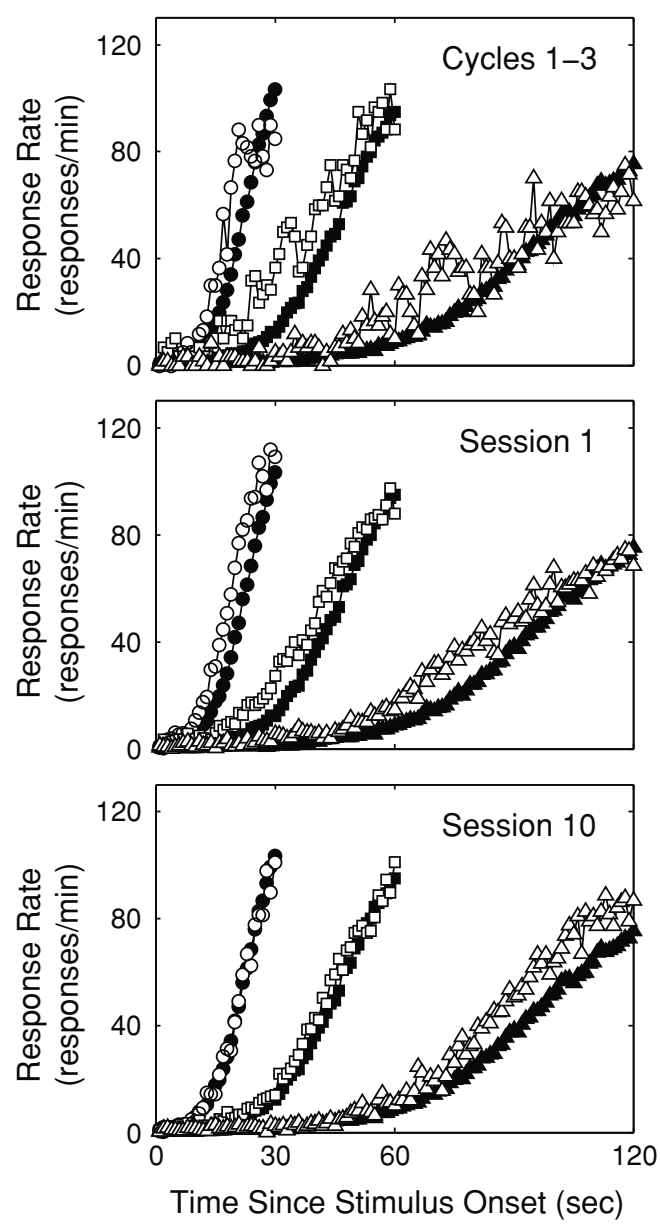

Figure 5. FI $30 \mathrm{sec}$ (circles), FI $60 \mathrm{sec}$ (squares), and FI $120 \mathrm{sec}$ (triangles). Response rate gradients for reacquisition (open symbols) is shown for Cycles 1-3 (top), Session 1 (middle), and Session 10 (bottom). The response rate gradients for the last 10 sessions in acquisition are shown in all three panels (filled symbols). 

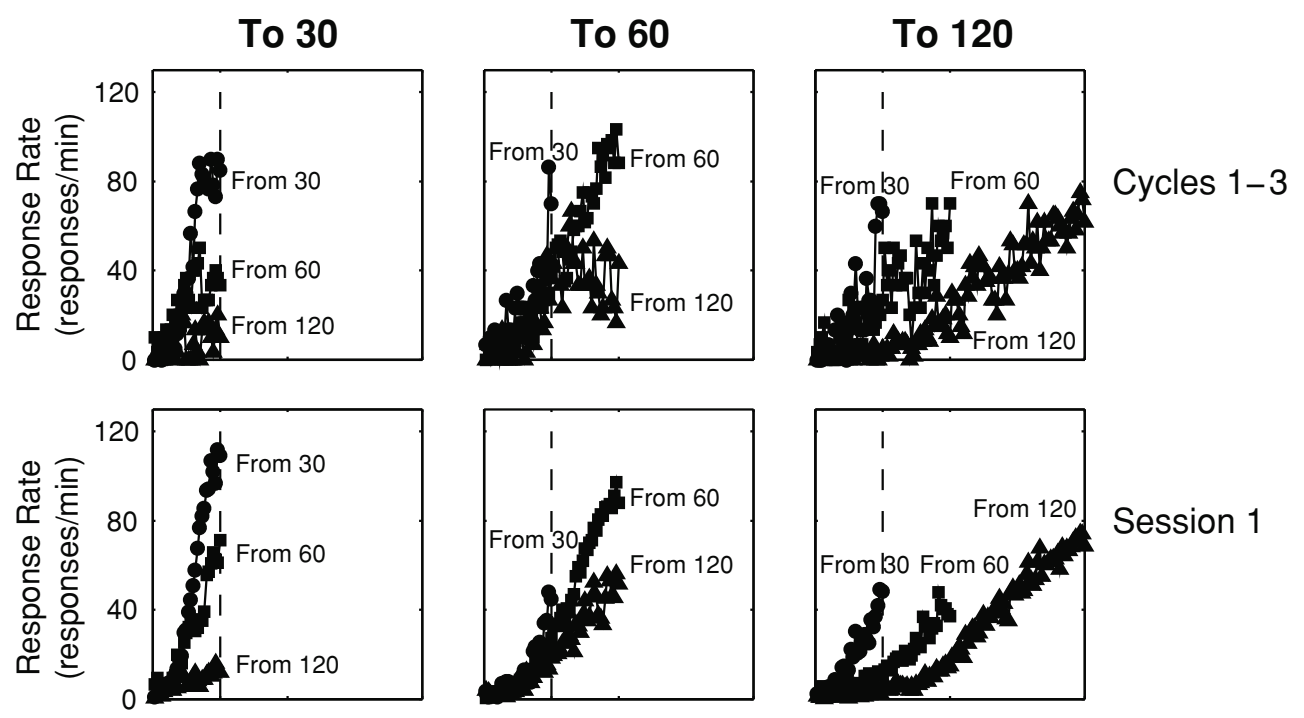

\section{Session 1}
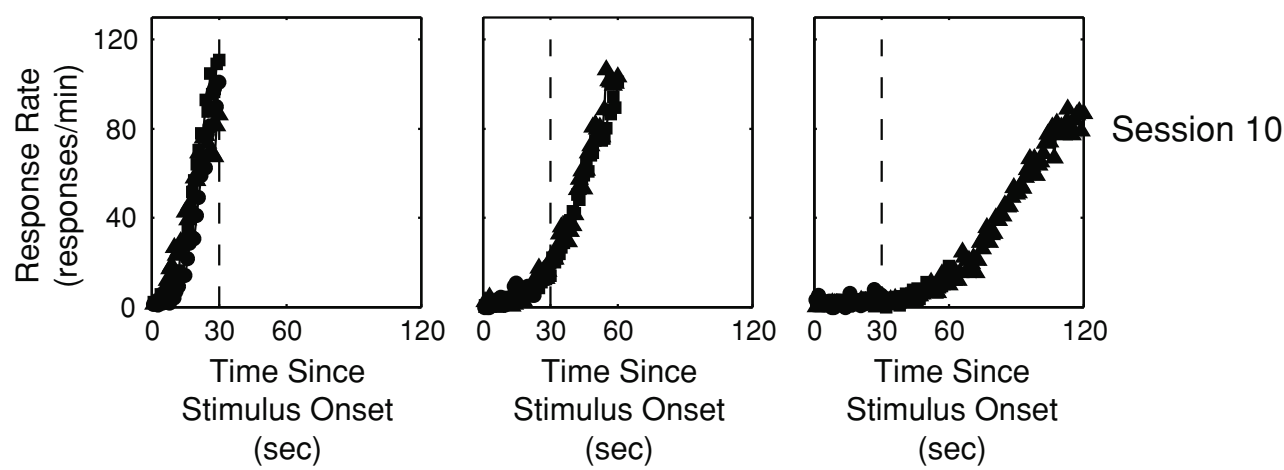

Figure 6. Response rate gradients during reacquisition that was consistent with acquisition (Group AAA, in which the "From interval" and the "To interval" are the same durations) and inconsistent with acquisition (Group AAB, in which the "From interval" and the "Io interval" are different durations). The data are shown for the first three cycles of reacquisition (top panels), the first session of reacquisition (center panels), and the last session of reacquisition (bottom panels). The interval trained in reacquisition was 30 sec (left panels), 60 sec (center panels), or 120 sec (right panels).

last session). In the first three cycles, the proportions of agreement of the response patterns with the conditions of original acquisition (A) exceeded the chance value of .50 $[t(11)=13.1, p<.001$, for Group AAA; and $t(11)=3.1$, $p<.01$, for Group AAB].

For Group AAA, the proportions of agreement were greater with the conditions of acquisition (A) than with a control condition (B) $[F(1,22)=435.0, p<.001]$, and there was no significant effect of reacquisition training or interaction. For Group AAB, there was a substantial interaction $[F(2,44)=14.6, p<.001]$. The control by conditions of reacquisition increased relative to the control by the conditions of original acquisition with reacquisition training.

\section{Discussion}

This experiment shows four main effects of extensive extinction of the head-entry response in a multiple cued interval procedure with rats: (1) a reduction in responding, (2) the maintenance of a low operant response rate, (3) the maintenance of temporal learning, and (4) an increase in the relative frequency of very short IRTs. A theory of extinction should be able to account for all of these results.

The most obvious effect of extinction is the reduction of responding, but the maintenance of a relatively constant daily pattern of responding even after 50 sessions of extinction requires explanation. During the last half of the 50 sessions of extinction, on a response scale appropriate for acquisition ( $0-50$ responses $/ \mathrm{min})$, the rate appeared to be about zero. In fact, as shown on a scale appropriate for extensive extinction ( $0-1$ response/min), some responses still occurred, at a rate slightly under 1 response in $2 \mathrm{~min}$. During the last half of each session, this rate appeared to be relatively constant. Thus, the asymptotic rate after extensive extinction remained at some low operant rate.

Two types of analyses were used to demonstrate the maintenance of temporal learning: one based on performance during extended extinction, and the other based on performance on a reacquisition transfer test. During extended extinction, the response gradients had the pattern of the response gradients during acquisition. In the re- 

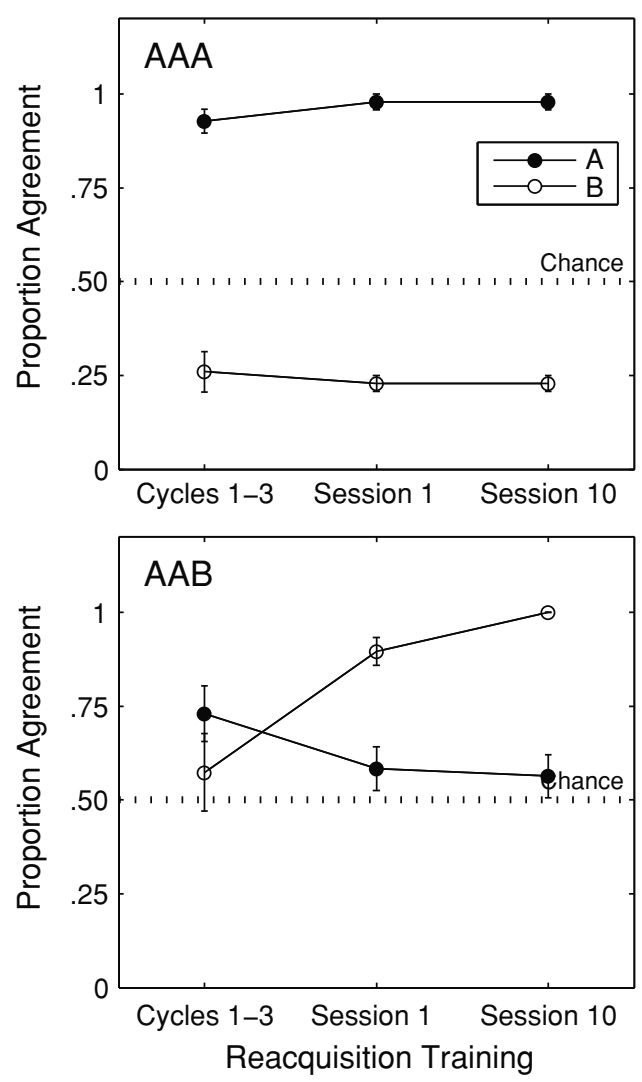

Figure 7. Proportion agreement as a function of amount of reacquisition training (first three cycles, first session, and last session) for the consistent group (Group AAA, top) and inconsistent group (Group AAB, bottom).

acquisition transfer test, the initial response gradients had the pattern of the response gradients during acquisition. This suggests that the temporal discriminations developed during acquisition were maintained during extinction and that this could be observed both during extinction and early in the reacquisition transfer test.

In both acquisition and extinction, responses occurred in bouts, with IRTs clustered under half a second. Most other responses were separated by more than $2.5 \mathrm{sec}$. In contrast to the response gradients that were closely related to the interval schedules, the distribution of IRTs within bouts were unaffected by the interval schedules $(30,60$, and $120 \mathrm{sec}$ ). They were, however, markedly different in extinction than in acquisition. Paradoxically, although extinction led to a large decrease in the overall rate of responding, the most frequent IRTs less than $2.5 \mathrm{sec}$ in extinction were shorter than those in acquisition.

\section{EXPERIMENT 2}

Experiment 1 showed that the memory for interval duration was preserved after extensive extinction. This was apparent in the response pattern during the last half of extinction and during the first three cycles of reacquisition with reinforcement that was consistent or inconsistent with original training. In Experiment 1, the durations of the stimuli during acquisition were the same as the durations of the stimuli during extinction (for both Groups $\mathrm{AAA}$ and $\mathrm{AAB}$ ). Thus, the conditions of acquisition and extinction were confounded: The response pattern for interval duration could have been preserved either because of retention of acquisition learning or because of the exposure to the interval durations during extinction.

The purposes of Experiment 2 were to replicate the four main effects of extensive extinction that were described in Experiment 1, and to determine whether the conditions of acquisition, extinction, or both influenced the rate and pattern of responding after extended extinction. The discussion will include some bases for explaining the reliable results.

As in Experiment 1, rats were trained under three discriminative fixed intervals $(30,60$, and $120 \mathrm{sec})$ signaled by different stimuli (light, noise, and clicker). After training, the rats were exposed to extensive extinction in which no food was delivered. The assignment of stimuli to intervals during extinction was either the same as, or different from, the assignment during acquisition. The pattern and initial rate of responding during the last half of the sessions of extinction were used to assess the effects of extended extinction on the rate and pattern of responding. After extinction, the rats were exposed to reacquisition of the temporal discrimination with the assignment of stimuli to intervals either the same as, or different from, assignments during acquisition and extinction.

The reduction of response rate of ring doves produced by extinction of autoshaped responding depends on the similarity of the stimulus durations during acquisition and extinction (Drew, Yang, Ohyama, \& Balsam, 2004). In Experiment 2 , we examined the effect of the similarity of the stimulus durations used in acquisition and extinction on the effects of extinction. The pattern of responding during the initial stages of the reacquisition was used to assess the pattern of responses after extended extinction. Moreover, they were used to determine whether acquisition, extinction, or both influenced the rate and pattern of responses.

\section{Method}

\section{Animals}

Twenty-four male Sprague Dawley rats were used in the experiment. Half of the animals were naive, and half had previous experience in an unrelated procedure in which food pellets were dropped in the food cup at either fixed or random times. The rats arrived in the colony at 35 days of age and were 55 (naive) or 137 (non-naive) days of age when the multiple cued interval training began. The treatment of the animals with respect to food, water, and handling were the same as in Experiment 1.

\section{Apparatus}

The apparatus in Experiment 2 was the same as that in Experiment 1 .

\section{Procedure}

The rats received the same training in the multiple cued interval procedure as did the animals in Experiment 1.

Phase 1: Acquisition (Sessions 1-30). The procedure of the acquisition phase was identical to the procedure used in the acquisi- 
tion phase of Experiment 1. Because, unlike in Experiment 1, the rats had no previous experience in this task, they were trained for 30 sessions. The assignment of stimuli (noise, light, and clicker) to intervals $(30,60$, or $120 \mathrm{sec})$ was counterbalanced across rats. One of the three possible intervals was presented randomly with equal probability on each of the 60 cycles of a daily session.

Phase 2: Extinction (Sessions 1-30). The second phase consisted of extinction of responses for all three intervals. Because the response rate was relatively constant and low after 30 of the 50 sessions of extinction in Experiment 1, 30 sessions of extinction were used. As in Experiment 1, at the end of the fixed interval, the stimulus was turned off and no food was delivered; head-entry responses into the food cup were recorded, but they had no consequences.

The stimuli associated with the three durations remained consistent with acquisition for half of the animals (as in Experiment 1), but they were inconsistent with acquisition for the other half. For example, with the inconsistent treatment, a rat that had noise, light, and clicker associated with the fixed intervals 30,60 , and $120 \mathrm{sec}$, respectively, during acquisition could have had the noise associated with the 60 -sec fixed interval, the light associated with the 120 -sec fixed interval, and the clicker associated with the 30 -sec fixed interval during extinction. The stimulus interval changes were counterbalanced across rats, and, in each condition, half of the rats were naive and half had previous experience.

Phase 3: Reacquisition (Sessions 1-12). The procedure in the reacquisition phase consisted of the delivery of reinforcement following the first head entry into the food cup after the 30-, 60-, or 120 -sec interval.

The experimental design is described in Table 2 for the four groups. As in Experiment 1, "A" refers to the assignment of stimuli to intervals (e.g., noise for FI $30 \mathrm{sec}$, light for FI $60 \mathrm{sec}$, and clicker for FI $120 \mathrm{sec}$ ). The two top rows consist of a direct replication of Experiment 1, in which Group AAA had the same assignment of stimuli to intervals during acquisition, extinction, and reacquisition, and Group $\mathrm{AAB}$ had the same assignment of stimuli to intervals during acquisition and extinction, but a different assignment (e.g., noise for FI $60 \mathrm{sec}$, light for FI $120 \mathrm{sec}$, and clicker for FI $30 \mathrm{sec}$ ) during reacquisition. The bottom two rows consist of two additional groups in which assignment of stimuli to intervals A occurred during acquisition and assignment of $\mathrm{B}$ during extinction. The difference between the two groups was in the assignment of $\mathrm{A}$ (third row) or $\mathrm{B}$ (bottom row) during reacquisition. These groups are referred to as Group ABA and Group ABB, respectively. Three naive rats and 3 rats with previous experience were randomly assigned to each of the four treatment conditions.

\section{Data Analysis}

The data analyses used in Experiment 2 were the same as used in Experiment 1.

\section{Results \\ Acquisition and Extinction}

The effects of stimulus duration $(30,60$, and $120 \mathrm{sec})$ on the overall response rate during acquisition are shown in the left side of the top panels of Figure 8 for Groups AAX (left panel) and ABX (right panel). For the statistical analysis, the overall response rates during the last 10 sessions of acquisition (Sessions 21-30) were averaged across rats from Groups AAX (left panel) and ABX (right panel), since both groups had the same treatment during acquisition (A). The overall response rates during the last 10 sessions of acquisition were 39.2, 41.9, and $36.2 \mathrm{responses} / \mathrm{min}$ during the $30-, 60-$, and 120 -sec intervals, respectively. Although the mean response rates were not inversely related to interval durations during acquisition, they were related to interval duration $[F(2,46)=$ $3.62, p<.05]$.

The effects of extinction on the overall response rate are shown in the right side of the top panels of Figure 8 for Group AAX (left) and Group ABX (right). The overall response rates decreased from the rates at the end of acquisition to less than 1 response/min. During the early sessions in extinction (first session), the overall response rates were inversely related to the duration of the stimulus presented during extinction for Group AAX, but not for Group ABX. This is shown by a significant interaction of stimulus duration $\times$ group $[F(2,44)=18.52, p<.001]$. In the first session of extinction, the overall response rates for the three intervals were inversely related to the stimulus durations presented during acquisition for Groups $\mathrm{AAX}$ and $\mathrm{ABX}[F(2,44)=49.84, p<.001]$, and the overall rate was higher for Group AAX than for Group ABX $[F(1,22)=6.11, p<.05]$. The stimulus duration $\times$ group interaction was not significant when the overall response rate during the first session in extinction was analyzed as a function of interval duration in acquisition $[F(1,22)=$ $0.52, p=.60]$.

During later sessions in extinction (last 15 sessions), the overall response rate appears to be close to zero in the top panels of Figure 8 . On a scale of $0-1$ response/min (bottom panels of Figure 8), however, the overall response rate during later sessions in extinction appears to be inversely related to the duration of the interval presented during extinction for Group AAX, but not for Group ABX. This is shown by a significant interaction of stimulus duration $X$ group $[F(2,44)=4.59, p<.05]$. The same stimulus duration $X$ group interaction was observed when the overall response rate during later sessions in extinction was analyzed as a function of stimulus duration in acquisition $[F(2,44)=3.55, p<.05]$. Therefore, the overall response rate during later sessions in extinction was inversely re-

Table 2

Design of Experiment 2

\begin{tabular}{lccc}
\hline \multicolumn{4}{c}{ Design of Experiment 2 } \\
\multicolumn{1}{c}{ Group } & $\begin{array}{c}\text { Phase 1 } \\
\text { Acquisition }\end{array}$ & $\begin{array}{c}\text { Phase 2 } \\
\text { Extinction }\end{array}$ & $\begin{array}{c}\text { Phase 3 } \\
\text { Reacquisition }\end{array}$ \\
\hline Consistent & $\mathrm{A}$ & $\mathrm{A}$ & $\mathrm{A}$ \\
Inconsistent (Phase 3) & $\mathrm{A}$ & $\mathrm{A}$ & $\mathrm{B}$ \\
Inconsistent (Phase 2) & $\mathrm{A}$ & $\mathrm{B}$ & $\mathrm{A}$ \\
Inconsistent (Phases 2 and 3) & $\mathrm{A}$ & $\mathrm{B}$ & $\mathrm{B}$ \\
\hline
\end{tabular}

Note-The first two rows of the design of Experiment 2 represent the same procedures as those used in Experiment 1. "A" designates a particular assignment of stimuli to intervals; "B" designates a different assignment. 

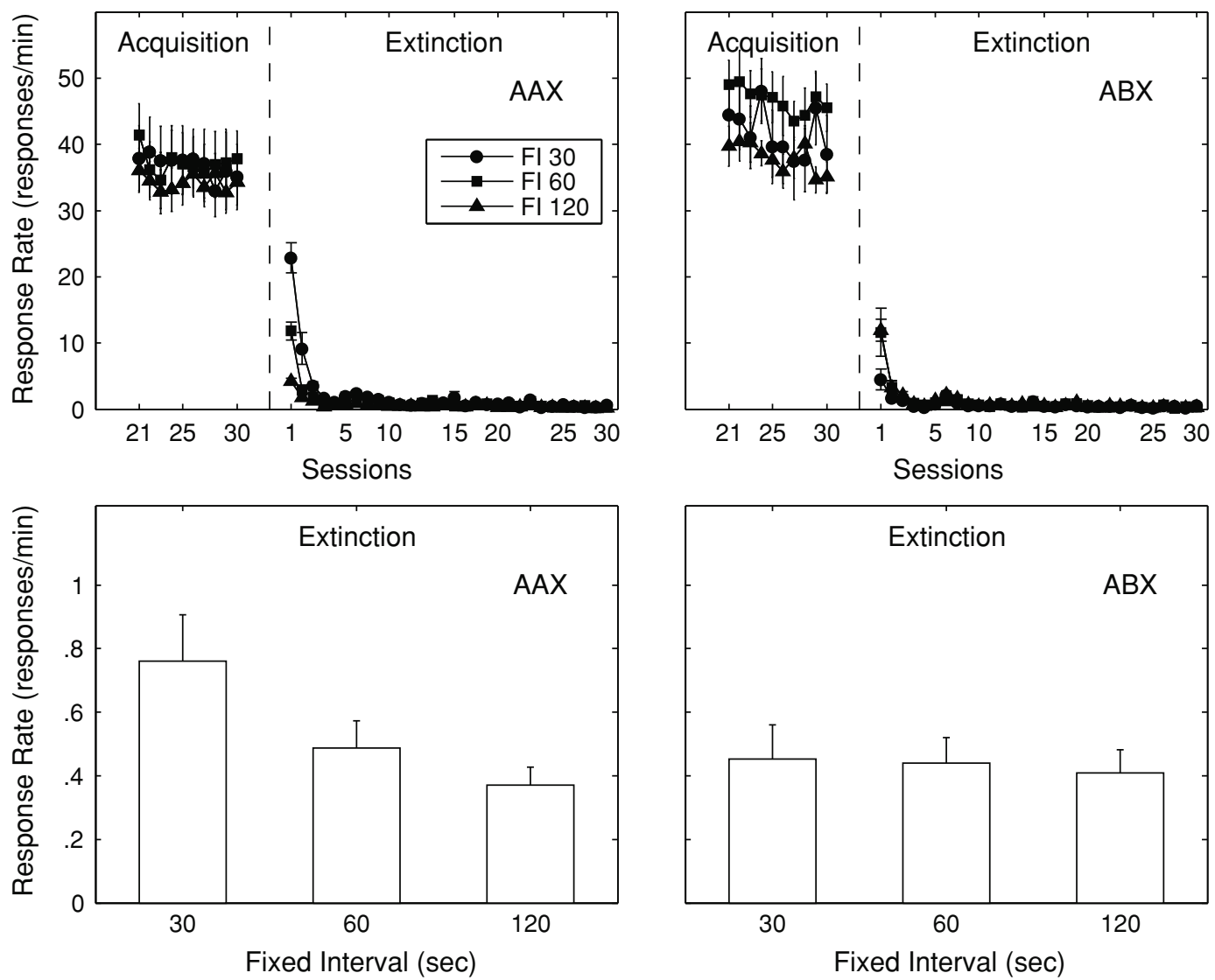

Figure 8. Top: Mean response rate during the stimuli as a function of sessions during the last 10 sessions of acquisition and during the 30 sessions of extinction during the 30-, 60-, and 120-sec fixed intervals. Bottom: Overall response rate averaged across the last 15 sessions of extinction as a function of fixed interval. The response rate scale in the bottom panel is $2 \%$ of the scale in the top panel.

lated to stimulus duration during both acquisition and extinction for Group AAX, and not related to stimulus duration in either acquisition or extinction for Group ABX.

The effects of the stimulus duration $(30,60$, and $120 \mathrm{sec}$ ) on the pattern of responses during acquisition are shown in the top panels of Figure 9 for Group AAX (left panel) and Group ABX (right panel). For statistical analysis, the response rate gradients during the last 10 sessions of acquisition (Sessions 21-30) were averaged across the rats from Groups AAX and ABX. The mean response gradients were approximately ogival functions that were related to stimulus duration and similar for Groups AAX and ABX. Although response rate is shown in absolute, rather than relative, units, the center and scale parameters of the ogive function were indicative of the pattern of responses. The proportion of variance accounted for by the ogive functions, averaged across rats and stimulus durations, was .990 and the interquartile range was .002 . The centers of the individual ogive functions were positively related to stimulus duration $[F(2,46)=287.8, p<.001]$ (means of 26.9, 41.3, and 81.9 at 30-, 60-, and 120-sec stimuli, respectively). The scales of the individual ogive functions were also positively related to the stimulus dura- tion $[F(2,46)=440.2, p<.001]$ (means of 4.5, 7.7, and 15.2 at $30-, 60-$, and 120 -sec intervals, respectively).

The response rate gradients during the early sessions in extinction (Session 1) were still related to the stimulus duration during acquisition for Groups $\mathrm{AAX}$ and $\mathrm{ABX}$ and related to extinction for Group AAX, but not Group ABX. The magnitude of the increase of the response rate functions of the individual rats was estimated by the slopes of a linear fit to the individual data. The mean slopes of the response rate gradients during the first session in extinction were $71.56,22.34$, and 3.30 for Group AAX, and $30.23,13.49$, and 1.98 for Group ABX, for the 30-, 60-, and 120 -sec stimulus durations in acquisition, respectively. These slopes were greater than zero $[t(23)=9.10$, $p<.001]$. These slopes were also related to the stimulus duration in acquisition $[F(2,46)=50.12, p<.001]$, more so for Group AAX than for Group $\mathrm{ABX}[F(1,22)=$ $19.32, p<.001]$. There was a stimulus duration $\times$ group interaction $[F(2,46)=9.31, p<.001]$.

In contrast, the slopes of the gradients were related to stimulus durations during the early sessions of extinction for Group AAX but not Group ABX. The mean slopes of the response rate gradients during the early sessions in ex- 

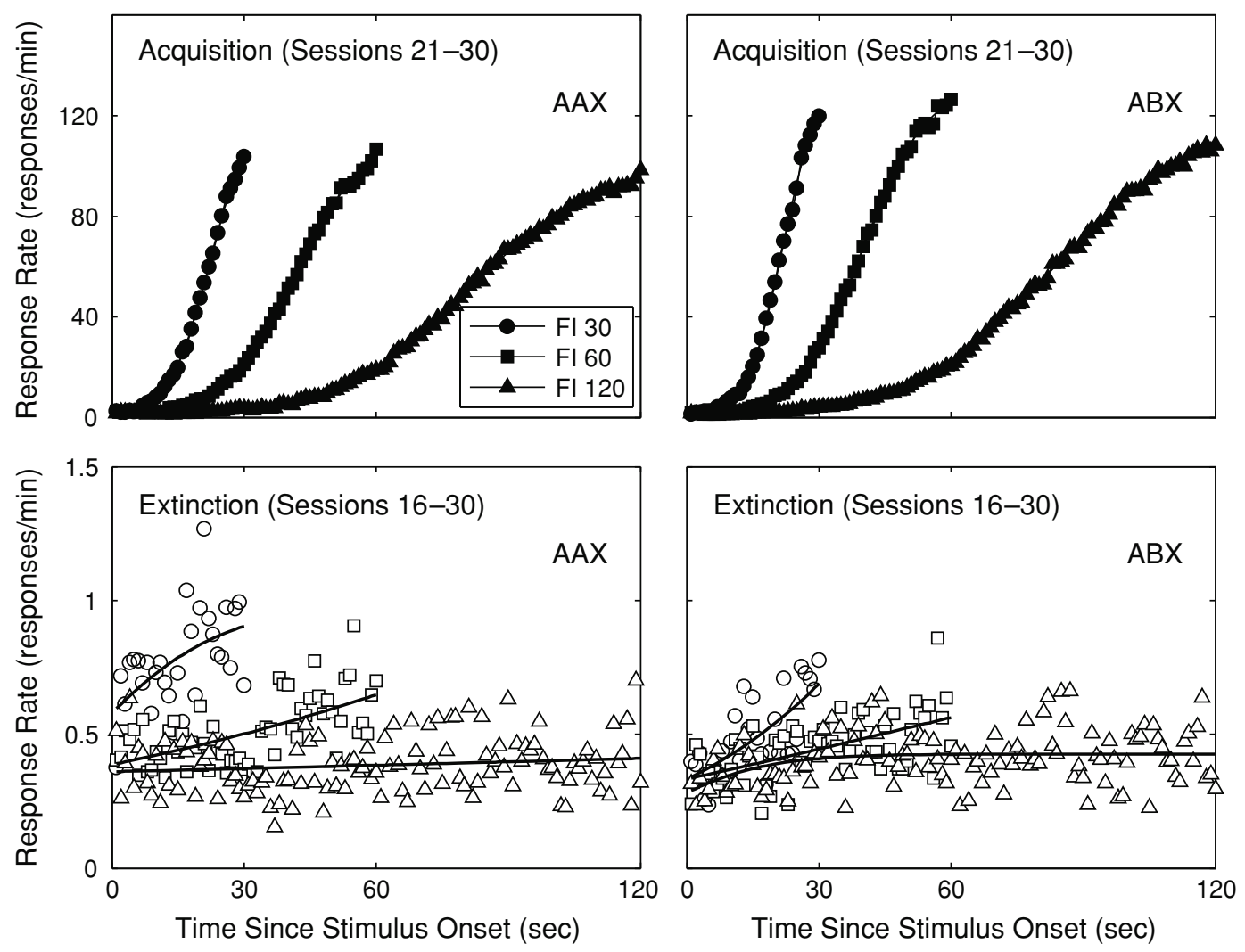

Figure 9. Response rate as a function of stimulus onset for the last 10 sessions of training (top) and last 15 sessions of extinction (bottom), during the fixed 30-, 60-, and 120-sec intervals for the consistent groups (AAX, left) and inconsistent group (ABX, right). The lines are the best-fitting ogives (Equation 1) to the data.

tinction were $10.72,15.31$, and 4.95 for Group $\mathrm{ABX}$, for the 30-, 60-, and 120-sec stimulus durations in extinction, respectively. This was shown by a significant stimulus duration during extinction $\times$ group interaction $[F(2,46)=$ $24.13, p<.001]$.

The effects of the duration of the stimulus during extinction $(30,60$, and $120 \mathrm{sec})$ on the pattern of responses during later sessions in extinction (last 15 sessions) are shown in the bottom panels of Figure 9 for Group AAX (left) and Group ABX (right). The response rate gradients during later sessions in extinction as a function of stimulus duration during extinction were related to the duration of the stimulus presented during extinction for Groups AAX and ABX. The lines shown amidst the averaged data are the best-fitting ogive (Equation 1) to the data. The degree of the increase of the response rate functions of the individual rats was estimated by the slopes of a linear fit to the individual data. These slopes of a linear fit to the response gradients (least squares criterion) were greater than zero $[t(23)=3.97, p<$ $.001]$ and were also related to the stimulus duration in extinction $[F(2,44)=5.28, p<.01]$. There was no difference between Group AAX and Group ABX $[F(1,22)=0.02, p=$ $.90]$, and there was no stimulus duration $\times$ group interaction $[F(2,44)=0.03, p=.98]$. The slopes for individual rats and means across rats during the 30-, 60-, and 120-sec stimuli are shown in Table 3 for rats in Groups AAX and ABX.
The results were somewhat different when response rate gradients were analyzed as a function of the stimulus duration during acquisition. Although the response rate gradients were related to stimulus duration for Group AAX, they were not related to stimulus duration for Group ABX. There was no effect of stimulus duration $[F(2,44)=.552, p=$ $.580]$ or group $[F(1,22)=0.17, p=.898]$, and the interaction was close, but not quite, significant $[F(2,44)=3.047$, $p=.058]$. Since the response rate gradients during later sessions in extinction for Group ABX were related to the stimulus duration in extinction, but not acquisition, there was an effect of extinction in the pattern of responding.

The ogival functions characteristic of the response patterns during acquisition and extinction started at an initial rate greater than zero during both acquisition and extinction. This initial operant response rate was measured as the rate during the first second after stimulus onset. It was not related to the duration of the stimulus presented during extinction $[F(2,44)=0.769, p=.470]$, and it was similar for Groups AAX and $\mathrm{ABX}[F(1,22)=0.337, p=$ .568]. The operant response rate was greater during acquisition than during extinction $[F(1,22)=6.827, p=$ $.016]$, but the magnitude of the difference was relatively small and there were no significant interactions. The mean initial rates during acquisition were 2.62, 2.54, and 1.80 for the 30-, 60-, and 120-sec stimuli, respectively, for 
Table 3

Slopes of a Linear Fit (Least Squares Criterion) to the Response Gradients of Individual Rats During the Last Half of Sessions of Extinction (Sessions 16-30)

\begin{tabular}{|c|c|c|c|}
\hline \multirow[b]{2}{*}{ Rats } & \multicolumn{3}{|c|}{ Fixed Intervals (sec) } \\
\hline & 30 & 60 & 120 \\
\hline \multicolumn{4}{|c|}{ Consistent (Group AAX) } \\
\hline 1524 & 2.21 & 0.41 & -0.06 \\
\hline 1528 & -0.53 & -0.06 & 0.09 \\
\hline 1536 & 1.43 & 0.87 & -0.05 \\
\hline 1576 & -0.35 & 0.58 & 0.04 \\
\hline 1583 & -1.77 & 0.15 & 0.18 \\
\hline 1587 & 1.92 & 0.50 & -0.08 \\
\hline 1526 & -0.24 & 0.35 & 0.01 \\
\hline 1531 & 0.34 & 0.04 & 0.02 \\
\hline 1534 & 0.72 & -0.09 & -0.05 \\
\hline 1580 & 1.32 & 0.13 & 0.10 \\
\hline 1585 & 0.29 & 0.39 & 0.01 \\
\hline 1586 & 2.24 & -0.26 & 0.06 \\
\hline Mean & 0.63 & 0.25 & 0.02 \\
\hline \multicolumn{4}{|c|}{ Inconsistent (Group ABX) } \\
\hline 1527 & -0.06 & 0.36 & 0 \\
\hline 1532 & 1.90 & -0.20 & 0.16 \\
\hline 1535 & -0.47 & 0.15 & -0.07 \\
\hline 1578 & -0.07 & -0.06 & -0.01 \\
\hline 1579 & 1.23 & -0.08 & -0.02 \\
\hline 1582 & 2.53 & 0.74 & -0.18 \\
\hline 1525 & 1.75 & 0.02 & 0.21 \\
\hline 1529 & -0.11 & -0.16 & 0.21 \\
\hline 1530 & 0.64 & -0.03 & -0.07 \\
\hline 1577 & 0.22 & 1.00 & -0.02 \\
\hline 1581 & -0.35 & 0.14 & 0.14 \\
\hline 1584 & 1.19 & 0.88 & 0.10 \\
\hline Mean & 0.70 & 0.23 & 0.04 \\
\hline
\end{tabular}

Group AAX, and 1.50, 2.01, and 1.69 for the 30-, 60-, and 120 -sec stimuli, respectively, for Group ABX. The mean initial rates during extinction were $0.38,0.41$, and 0.51 for the 30-, 60-, and 120-sec intervals, respectively, for Group AAX, and 0.40, 0.24, and 0.32 for the 30-, 60-, and 120 -sec intervals, respectively, for Group ABX.

The decrease in the overall response rate within a session during extinction is shown in Figure 10. The data are represented as the mean of the response rates averaged across stimulus durations and individual rats during the last 15 sessions of extinction. The decrease was similar at all three intervals $(30,60$, and $120 \mathrm{sec})$, and the response rate was higher in Cycle 1 than in Cycle $15[t(23)=$ $11.20, p<.001]$. As in Experiment 1, although the response rate at the beginning of a session was greater than the response rate at the end of the session, responses still occurred late in a session. The response rates during $\mathrm{Cy}-$ cles $11-15$ of the last 15 sessions of extinction were similar at all intervals: the response rates were $0.30,0.22$, and 0.23 response $/ \mathrm{min}$, and the standard errors of the means were $0.06,0.04$, and 0.03 , for the fixed 30-, 60-, and 120 sec intervals, respectively.

The IRT distributions were not affected by the interval duration but were affected by extinction. The relative frequencies of IRTs ranging from 0 to $2.5 \mathrm{sec}$ during the last 10 sessions of acquisition and last 15 sessions of ex- tinction are shown in Figure 11. The means of the modes of the IRT distributions across intervals were shorter during extinction than during acquisition $[t(23)=4.72, p<$ $.001]$. The mean modes of the IRT distributions averaged across rats and intervals were $0.25 \mathrm{sec}$ during acquisition and $0.14 \mathrm{sec}$ during extinction, with the standard errors of the means approximately $0.02 \mathrm{sec}$ during acquisition and extinction. Thus, the IRTs were about $0.11 \mathrm{sec}$ longer during acquisition than during extinction.

During both acquisition and extinction, the IRTs from 0 to $2.5 \mathrm{sec}$ clustered below $0.5 \mathrm{sec}$. During acquisition, these short IRTs accounted for $92.9 \%, 93.8 \%$, and $93.3 \%$ of the IRTs in the 30-, 60-, and 120-sec intervals, respectively; during extinction, these short IRTs accounted for $53.1 \%$, $54.4 \%$, and $53.6 \%$ of the IRTs in the $30-, 60-$, and 120 -sec intervals, respectively. During acquisition, the standard error of the means were $0.89 \%, 0.86 \%$, and $0.75 \%$ in the $30-, 60-$, and 120 -sec intervals, respectively; during extinction, they were $5.03 \%, 2.95 \%$, and $2.41 \%$ in the $30-, 60-$, and 120 -sec intervals, respectively. The longer IRTs during acquisition were quite distinct from the shorter IRTs during extinction.

\section{Reacquisition}

The overall response rate during the stimulus in reacquisition rapidly increased (Cycles 1-3) from the low rates at the end of extinction to a rate similar to that at the end of acquisition.

During the first $30 \mathrm{sec}$ of each of the stimuli $(30,60$, and $120 \mathrm{sec}$ ), the relative response rate was inversely related to the interval. (See the first $30 \mathrm{sec}$ of the gradients in Figure 9, as well as the description of the "proportion agreement" measure in the data analysis section of Experiment 1.) Figure 12 shows the proportion agreement of the observed order of the relative response rate during the first $30 \mathrm{sec}$ of the $30-, 60-$, and 120 -sec stimuli to the expected order, based on the assignments of stimuli to intervals used in original acquisition (A) or on the assignments of stimuli to intervals used in some groups

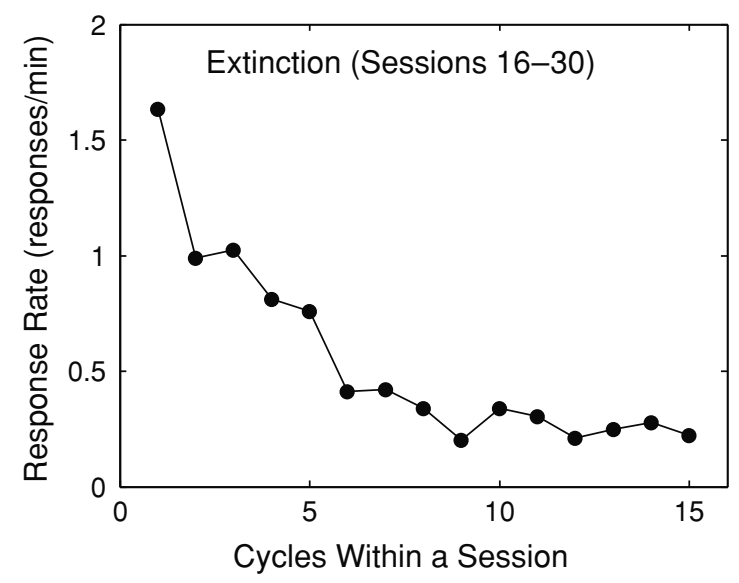

Figure 10. Response rate as a function of the first 15 cycles within a session averaged over the last 15 sessions of extinction and averaged across the fixed 30-, 60-, and 120-sec intervals. 


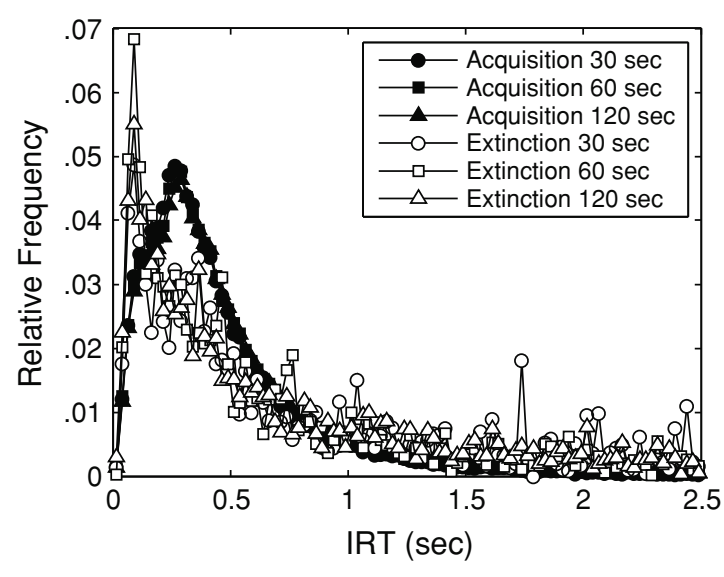

Figure 11. Relative frequency of interresponse intervals (IRTs) ranging from 0 to $2.5 \mathrm{sec}$ during the last 10 sessions of acquisition (filled symbols) and last 10 sessions of extinction (open symbols) during the fixed 30-, 60-, and 120-sec intervals.

during extinction and/or retraining. The horizontal axis represents the amount of reacquisition training (first three cycles, first session, or last session). In the first three cycles, the proportion of agreement of the response patterns with the conditions of original acquisition (A) exceeded the chance value of .50 for three of the four groups. The rats in Groups AAA, ABA, and ABB had agreement with pattern $A$ above chance $[t(5)>6.3, p<.002]$, but for the rats in Group AAB, the proportion agreement with pattern A was not significantly greater than chance.

The top two panels of Figure 12 show the conditions of reacquisition consistent with the conditions of acquisition (AAA and ABA); here it can be seen that the proportion of agreement with the conditions of acquisition (A) was greater than the proportion of agreement with the control condition (B) $[F(1,10)=288.0, p<.001$, and $F(1,10)=$ $92.9, p<.001]$. In the bottom two panels, which show the conditions of reacquisition inconsistent with the conditions of acquisition (AAB and $\mathrm{ABB}$ ), it can be seen that there was a substantial interaction $[F(2,44)=24.4, p<.001$; $F(2,20)=29.1, p<.001]$. As in Experiment 1, the control by the conditions of reacquisition gradually increased relative to the control by the pattern of original acquisition.

\section{Discussion}

The treatment of two of the four groups (AAA and $\mathrm{AAB}$ ) was the same as that for the two groups in Experiment 1 ; the treatment of the other two groups during extinction was different (ABA and $\mathrm{ABB})$.
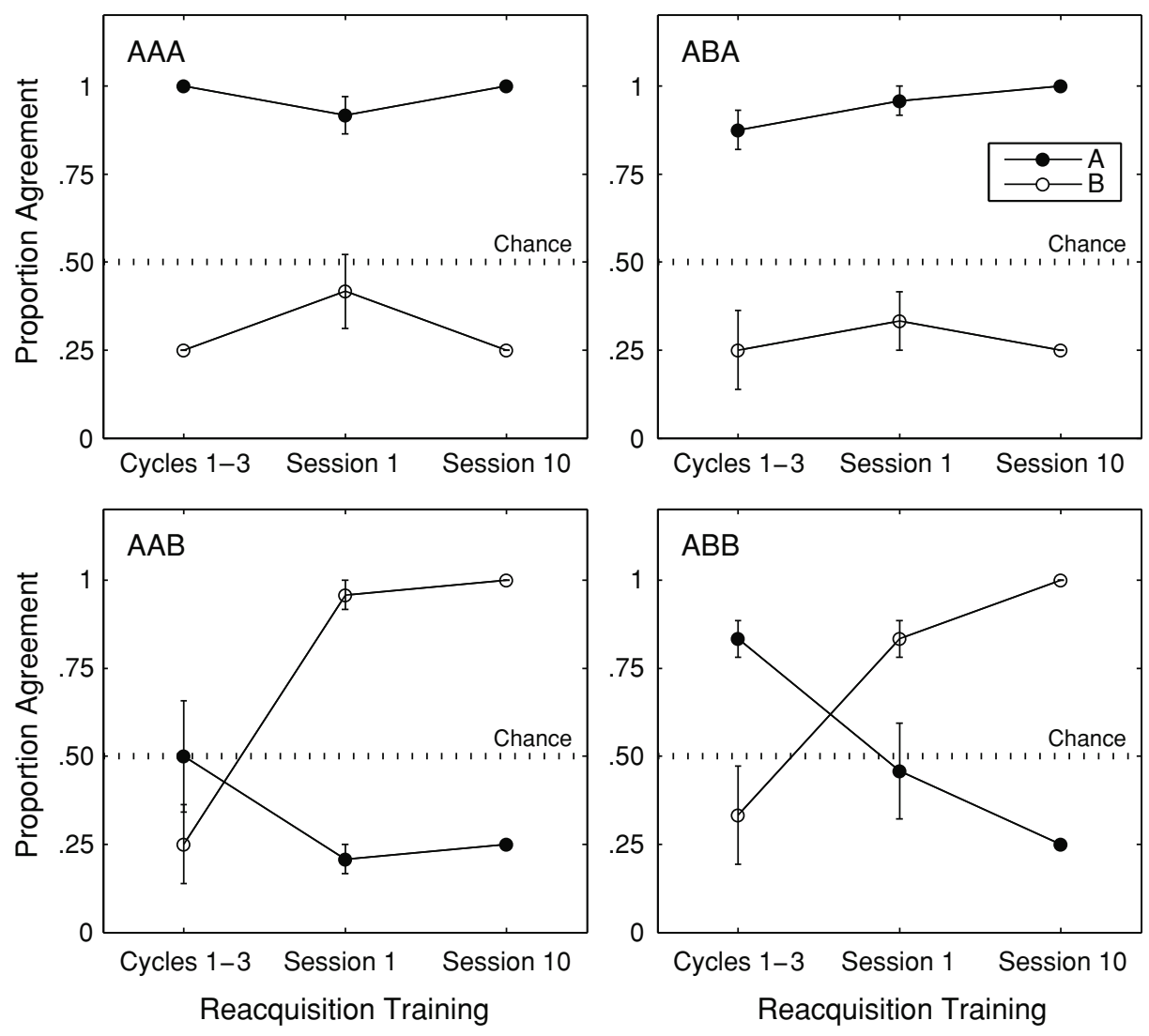

Figure 12. Proportion agreement as a function of amount of reacquisition training (first three cycles, first session, and last session) for the consistent group (Group AAA, top left) and inconsistent groups (Group AAB, bottom left; Group ABA, top right; and Group ABB, bottom right). 
The purpose of testing Groups AAA and AAB was to replicate the essential results of Experiment 1, and to provide a control group for the assessment of the effect of the treatment during extinction. These conditions replicated the four main effects of extensive extinction described in Experiment 1: (1) a reduction in responding, (2) the maintenance of a low operant response rate, (3) the maintenance of temporal learning, and (4) an increase in the relative frequency of very short IRTs.

The purpose of testing Groups ABA and ABB was to assess the effect of the stimulus durations during extinction. The gradients during extinction were related to the stimulus duration during extinction for Groups AAX and ABX. Thus, extinction changed the pattern of responses when the interval durations in extinction were not consistent with the stimulus durations trained in acquisition. Nonetheless, during the first three cycles of reacquisition, the response gradients of all were related to the stimulus durations trained during acquisition. The pattern of responding at the three fixed intervals that was learned during acquisition was maintained throughout the extensive extinction, although, for Group ABX, this maintenance was not directly shown in later sessions during extinction.

The results are summarized in Table 4. The pattern of gradients in later sessions during acquisition was related to the treatment during acquisition (A). During early cycles in extinction, the same pattern (A) was maintained; but during later sessions in extinction, the pattern changed from the pattern trained in acquisition (A) to the treatment used in extinction (A or B). This is shown in the columns under Extinction in Table 4. For example, in Group ABX, a rat with an acquisition treatment of light $-30 \mathrm{sec}$, noise$60 \mathrm{sec}$, and clicker-120 sec might have an extinction treatment of clicker-30 sec, light-60 sec, and noise- $-120 \mathrm{sec}$. Early in extinction, such a rat would have the pattern of responses characteristic of the conditions of acquisition (A), but during later sessions in extinction, the rat would have the pattern of responses characteristic of the conditions of extinction (B). A plausible interpretation of this result is that, in acquisition, the rats learned the intervals between stimulus onset and food (light-30 sec, noise- $60 \mathrm{sec}$, and clicker-120 sec), stimulus onset and termination (also light-30 sec, noise-60 sec, and clicker-120 sec), and stimulus termination and food $(0 \mathrm{sec})$; in extinction, they learned the new intervals between stimulus onset and termination. The pattern in extinction reflected this new learning, and because early in extinction the rats in the Group ABX still showed a pattern that was related to the acquisition intervals rather than the extinction intervals, the pattern late in extinction was not simply a performance due to the changes in the stimulus durations.

During the early cycles in reacquisition, the pattern reverted to the pattern in acquisition (rather than extinction). This is presumably an example of context learning in which the reinforcer may have acted as a discriminative stimulus that, during reacquisition, restored the pattern of responding learned during acquisition. The context of reacquisition, with food deliveries, is the same as the context of acquisition. Thus, the performance in reacquisition is the same as the performance in acquisition. Of course, the pattern during later sessions in reacquisition reflected the treatment conditions in reacquisition (A or B). The effects of treatment on response pattern during the early cycles and later sessions of reacquisition are shown in the columns under Reacquisition in Table 4.

These results supported the conclusion that extinction reduces response rate, but that it does not eliminate the content learned during acquisition. In the standard conditioning procedure, in which the conditions of extinction are the same as those during acquisition except for the delivery of reinforcers, the content may be observed throughout extensive extinction and during early cycles in reacquisition (with both consistent and inconsistent treatments). Learning of stimulus duration in extinction may be observed during later sessions in extinction, but this did not eliminate the content of learning in acquisition. When the original context of food delivery was restored in reacquisition, the initial pattern of responding reflected the performance during acquisition, not during extinction.

To explain the four main effects of extensive extinction shown in both Experiments 1 and 2, and the one additional effect shown in Experiment 2, a theory of extinction should probably include the following features: (1) To account for the rate of responding, it should include a standard strength memory that increases with reinforcement and decreases with nonreinforcement, and a baseline operant rate; (2) to account for the pattern of responding, it should also include a pattern memory that is influenced by the time from onset of a stimulus until reinforcement; and (3) to account for the IRT distribution during acquisition and extinction, it should include the concept of response packets.

Table 4

Effect of Treatment on Response Pattern in Acquisition, Extinction, and Reacquisition

\begin{tabular}{|c|c|c|c|c|c|c|c|c|c|}
\hline \multirow[b]{3}{*}{ Group } & \multicolumn{3}{|c|}{ Acquisition } & \multicolumn{3}{|c|}{ Extinction } & \multicolumn{3}{|c|}{ Reacquisition } \\
\hline & \multirow[b]{2}{*}{ Treatment } & \multicolumn{2}{|c|}{$\begin{array}{c}\text { Response } \\
\text { Pattern }\end{array}$} & \multirow[b]{2}{*}{ Treatment } & \multicolumn{2}{|c|}{$\begin{array}{c}\text { Response } \\
\text { Pattern }\end{array}$} & \multirow[b]{2}{*}{ Treatment } & \multicolumn{2}{|c|}{$\begin{array}{c}\text { Response } \\
\text { Pattern }\end{array}$} \\
\hline & & Early & Late & & Early & Late & & Early & Late \\
\hline AAA & A & - & A & A & A & A & A & A & $\mathrm{A}$ \\
\hline $\mathrm{AAB}$ & A & - & A & A & A & A & $\mathrm{B}$ & A & B \\
\hline $\mathrm{ABA}$ & A & - & A & B & A & B & A & A & A \\
\hline $\mathrm{ABB}$ & A & - & A & B & A & B & B & A & B \\
\hline
\end{tabular}

Note- "Early" refers to the first three cycles. "Late" refers to the last half of the sessions of each phase. 
Some theories, such as those that feature the use of a linear operator rule (Bush \& Mosteller, 1955; Rescorla \& Wagner, 1972) provide a way of accounting for many factors affecting response strength. The Rescorla-Wagner model has been extensively used because of its ability to predict competitive effects between associations. The addition of a simple linear response rate rule to the Rescorla-Wagner model has been shown to be sufficient to account for the mapping of response rate into response strength during successive acquisitions and extinctions of fixed intervals (Guilhardi, Yi, \& Church, 2006). It does not, however, account for the pattern of responding.

In contrast, scalar timing theory (Gibbon, 1977, 1991; Gibbon, Church, \& Meck, 1984) has been used extensively to predict the pattern of behavior. It includes a memory that contains the examples of remembered times of reinforcement. It does not account for response strength. It has, however, been combined with rate expectancy theory to provide predictions of both pattern and rate (Gallistel \& Gibbon, 2000).

Other theories that predict response rate (strength) and response pattern (content) during extinction have been developed. For example, the learning to time theory (Machado, 1997) has been used to predict the pattern and rate of responding during fixed interval schedules during reinforcement and extinction (Machado \& Cevik, 1998).

Most current theories provide the basis for predicting particular summary statistics (such as mean response rate), but not the primary (such as the times of responses). From a theory that produced primary data, it would be possible to predict any summary statistics. The concept of response packets is helpful in the explanation of the IRT distribution during acquisition and extinction (Guilhardi, Keen, MacInnis, \& Church, 2005; Kirkpatrick, 2002; Kirkpatrick \& Church, 2003). A bout of head entry responses may be defined as a sequence of responses separated by less than $2.5 \mathrm{sec}$. This leads to the paradoxical fact that bout rate is faster in extinction than in acquisition. But if a distinction is made between a theoretical packet of response with well-defined characteristics and an observed bout of responses, as defined above, the paradox may be resolved as follows: In extinction, packets occur at a much lower rate than during acquisition. Typically, in extinction, the rate of packets is low enough that each packet produces a single bout. In contrast, in acquisition, the rate of packets is high enough that a bout may consist of several packets. Thus, with a refractory period between successive packets of about $110 \mathrm{msec}$, the relative frequency distribution of IRTs would be about $110 \mathrm{msec}$ longer in acquisition (with multiple packets) than in extinction (with normally a single packet). If, however, all IRTs are considered, there are many more IRTs in acquisition than in extinction and the mean of all IRTs is greater in extinction than in acquisition. They can be seen by examining the frequency distribution with logarithmic (rather than linear) spacing of intervals (Tolkamp \& Kyriazakis, 1999).

A theory of extinction also should account for the learning of stimulus duration without reinforcement during extinction, and for discrimination learning based on context. With these factors, it may be possible to understand the effect of extinction on reducing response rate, and on controlling the pattern of responding during extinction without changing performance during early cycles in reacquisition.

A quantitative theory adequate to explain the four main effects of extensive extinction will undoubtedly include a combination of ideas that are currently used in conditioning and timing models, and some others.

\section{REFERENCES}

Bush, R. R., \& Mosteller, F. (1955). Stochastic models for learning. New York: Wiley.

Church, R. M., \& Black, A. H. (1958). Latency of the conditioned heart rate as a function of the CS-US interval. Journal of Comparative \& Physiological Psychology, 51, 478-482.

Coleman, S. R., \& Gormezano, I. (1971). Classical conditioning of the rabbit's nictitating membrane response under symmetrical CS-US interval shifts. Journal of Comparative \& Physiological Psychology, 77, 447-455.

Delamater, A. R. (1996). Effects of several extinction treatments upon the integrity of Pavlovian stimulus-outcome associations. Animal Learning \& Behavior, 24, 437-449.

Drew, M. R., Yang, C., Ohyama, T., \& Balsam, P. D. (2004). Temporal specificity of extinction in autoshaping. Journal of Experimental Psychology: Animal Behavior Processes, 30, 163-176.

Gallistel, C. R., \& Gibbon, J. (2000). Time, rate, and conditioning. Psychological Review, 107, 289-344.

GibBon, J. (1977). Scalar expectancy theory and Weber's law in animal timing. Psychological Review, 84, 279-325.

GibBon, J. (1991). Origins of scalar timing theory. Learning \& Motivation, 22, 3-38.

Gibbon, J., Church, R. M., \& Meck, W. H. (1984). Scalar timing in memory. In J. Gibbon \& L. Allan (Eds.), Timing and time perception (Annals of the New York Academy of Sciences, Vol. 423, pp. 52-77). New York: New York Academy of Sciences.

Guilhardi, P., \& Church, R. M. (2005). Dynamics of temporal discrimination. Learning \& Behavior, 33, 399-416.

Guilhardi, P., Keen, R., MacInnis, M. L. M., \& Church, R. M. (2005). How rats combine temporal cues. Behavioural Processes, 69, 189-205.

Guilhardi, P., Yi, L., \& Church, R. M. (2006). Effects of repeated acquisitions and extinctions on response rate and pattern. Journal of Experimental Psychology: Animal Behavior Processes, 32, 322-328.

Hilgard, E. R., \& Marquis, D. G. (1940). Conditioning and learning. New York: Appleton-Century.

Hull, C. L. (1943). Principles of behavior. New York: Appleton-Century.

KAMIN, L. J. (1954). Traumatic avoidance learning: The effects of CSUS interval with a trace-conditioning procedure. Journal of Comparative \& Physiological Psychology, 47, 65-72.

Kimble, G. A. (1961). Hilgard and Marquis' conditioning and learning. New York: Appleton-Century-Crofts.

KIrkPatrick, K. (2002). Packet theory of conditioning and timing. Behavioural Processes, 57, 89-106.

Kirkpatrick, K., \& Church, R. M. (2003). Tracking of the expected time to reinforcement in temporal conditioning procedures. Learning \& Behavior, 31, 3-21.

Machado, A. (1997). Learning the temporal dynamics of behavior. Psychological Review, 104, 241-265.

Machado, A., \& CeviK, M. (1998). Acquisition and extinction under periodic reinforcement. Behavioural Processes, 44, 237-262.

Mackintosh, N. J. (1974). The psychology of animal learning. London: Academic Press.

Nevin, J. A. (1967). Effects of reinforcement scheduling on simultaneous discrimination performance. Journal of the Experimental Analysis of Behavior, 10, 251-260.

Ohyama, T., Gibbon, J., Deich, J. D., \& Balsam, P. D. (1999). Temporal control during maintenance and extinction of conditioned keypecking in ring doves. Animal Learning \& Behavior, 27, 89-98.

Pavlov, I. P. (1927). Conditioned reflexes (G. V. Anrep, Trans.). London: Oxford University Press. 
Rescorla, R. A. (1996). Preservation of Pavlovian associations through extinction. Quarterly Journal of Experimental Psychology, 49B, 245258.

Rescorla, R. A. (2001). Experimental extinction. In S. B. Klein \& R. R. Mowrer (Eds.), Handbook of contemporary learning theories (pp. 119-154). Mahwah, NJ: Erlbaum.

Rescorla, R. A., \& WAGNer, A. R. (1972). A theory of Pavlovian conditioning: Variation in the effectiveness of reinforcement and nonreinforcement. In A. H. Black \& W. F. Prokasy (Eds.), Classical conditioning II (pp. 64-99). New York: Appleton-Century-Crofts.

Roberts, S., \& Holder, M. D. (1984). What starts an internal clock? Journal of Experimental Psychology: Animal Behavior Processes, 10, 273-296.

SCHNEIDER, B. A. (1969). A two-state analysis of fixed-interval responding in the pigeon. Journal of the Experimental Analysis of Behavior, 12, 677-687.

TAтHAM, T. A., \& Zurn, K. R. (1989). The MED-PC experimental apparatus programming system. Behavior Research Methods, Instruments, \& Computers, 21, 294-302.

TolKamp, B. J., \& KYRIAZAKIS, I. (1999). To split behaviour into bouts, log-transform the intervals. Animal Behaviour, 57, 807-817.

\section{ARCHIVED MATERIALS}

The following materials associated with this article may be accessed through the Psychonomic Society's Norms, Stimuli, and Data archive, www.psychonomic.org/archive.

To access these files, search the archive for this article using the journal (Learning \& Behavior), the first author's name (Guilhardi) and the publication year (2006).

FILE: Experiment-1-Acquisition.zip

DESCRIPTION: The compressed archive file contains .txt data files (480) for all Acquisition sessions for all 24 rats in Experiment 1. The primary data are the times (column 1) of events (column 2) that occurred during the experimental session, such as the times of responses, food deliveries, and onset and termination of stimuli. A readme file is also contained (readme.pdf). The readme file contains a description of the supplementary material, such as content, file formats, and file naming conventions.

FILE: Experiment-1-Extinction.zip

DESCRIPTION: The compressed archive file contains .txt data files $(1,200)$ for all Extinction sessions for all 24 rats in Experiment 1. The primary data are the times (column 1) of events (column 2) that occurred during the experimental session. A readme file is also contained (readme .pdf). The readme file contains a description of the supplementary material, such as content, file formats, and file naming conventions.

FILE: Experiment-1-Reacquisition.zip

DESCRIPTION: The compressed archive file contains .txt data files (240) for all Reacquisition sessions for all 24 rats in Experiment 1. The primary data are the times (column 1) of events (column 2) that occurred during the experimental session. A readme file is also contained (readme .pdf). The readme file contains a description of the supplementary material such as content, file formats, and file naming conventions.

FILE: Experiment-2-Acquisition.zip

DESCRIPTION: The compressed archive file contains .txt data files (720) for all Acquisition sessions for all 24 rats in Experiment 2. The primary data are the times (column 1) of events (column 2) that occurred during the experimental session. A readme file is also contained (readme.pdf). The readme file contains a description of the supplementary material, such as content, file formats, and file naming conventions.

FILE: Experiment-2-Extinction.zip

DESCRIPTION: The compressed archive file contains .txt data files (720) for all Extinction sessions for all 24 rats in Experiment 2. The primary data are the times (column 1) of events (column 2) that occurred during the experimental session. A readme file is also contained (readme.pdf). The readme file contains a description of the supplementary material, such as content, file formats, and file naming conventions.

FILE: Experiment-2-Reacquisition.zip

DESCRIPTION: The compressed archive file contains .txt data files (288) for all Reacquisition sessions for all 24 rats in Experiment 2. The primary data are the times (column 1) of events (column 2) that occurred during the experimental session. A readme file is also contained (readme .pdf). The readme file contains a description of the supplementary material, such as content, file formats, and file naming conventions.

AUTHORS'E-MAIL ADDRESSES: paulo_guilhardi@brown.edu, russell_ church@brown.edu.

AUTHOR's Web SITE: www.brown.edu/Research/Timelab.

(Manuscript received August 16, 2005 revision accepted for publication February 13, 2006.) 